Introduction Sao Paulo (SP) state has 43 million inhabitants, 645 cities, 251.133 reported AIDS cases and a $0.6 \% \mathrm{HIV}$ prevalence. In SP 4 in 10 HIV diagnosed individuals' access care with delay.

Methods Conducting state testing campaigns annually is one of its main strategies to expand the offer of testing in the primary care health services especially in medium and small municipalities, besides the offer to pregnant women. The goal is to increasing the proportion of people tested once in life from $39.7 \%$ (2004) to $80 \%$ in 2017.

Results Approximately 3 million tests, including HIV and syphilis, were conducted in nine annual testing campaigns, of which 60\% are HIV tests and 40\% syphilis tests since 2014. An average of 130,000 HIV tests of the public health sector are billed in monthly. The number of municipalities involved rose from $376(58 \%)$ to $576(89 \%)$ between 2008/2016. Some results are very similar in all campaigns. The proportion of people tested for the first time revolves around 50\%, being always highest among males. The proportion of HIV positive identified is also quite stable and is around $0.45 \%$. By introducing rapid testing initially this strategy reached the level of $1.7 \%$ of HIV positive cases in 2010, gradually falling until 2016, reaching $0.4 \%$. The campaigns implementation in all nine years included a preparatory phase pacts in areas of management meetings and incorporated strategies for distance education and subsequent on-line monitoring.

Conclusion The HTC have amplified the access to HIV tests due to the expansion of spontaneous demand for testing services in primary care, especially in medium and small towns. Besides, campaigns arrangements are improving greater integration of primary care and STD/AIDS health programs, contributing for reducing the stigma related to AIDS and HIV testing in the population. Other complementary HIV testing strategies focused on vulnerable populations, conducted by health professionals, are easier to implement when already implemented in services routines.

\section{P3.118 PREVALENCE AND CORRELATES OF SEXUALLY TRANSMITTED INFECTION AMONG HOMELESS PERSONS IN CENTRAL BRAZIL}

Karlla Antonieta Amorim Caetanom; Matos MA, SA Teles, RS Pinheiro, PMRS Santos, MM Souza, ALN Junqueira, NPS Silva, JP Souza, ADL Guerra, MA Matos. Federal University of Goiás, Goiânia - GO, Brazil

\subsection{6/sextrans-2017-053264.353}

Introduction Current trends in Sexually Transmitted Infection (STI) epidemics indicate a shift in their profiles, with accelerated growth in more vulnerable populations such as people living on the streets. The objective of this study was to estimate the prevalence of STIs and to evaluate the risk behaviours for these infections among homeless persons in Goiânia, Central Brazil.

Methods This cross-sectional study was conducted between August 2014 and June 2015. Eligible participants were homeless persons sheltered in public housing of Goiânia, in Central Brazil, aged 18 years or older. All were interviewed on demographics, history of street living, sexual behaviour, and substance use. Blood samples were submitted to rapid tests for HIV, syphilis, and hepatitis $\mathrm{B}$, and a positive result on any one or more of these tests indicated presence of an STI. Univariate and multivariable Poisson regressions were done to identify correlates of ISTs. This study was approved by the Research Ethics Committee of the Federal University of Goiás. Results Among 353 eligible homeless persons, the majority were male $(81.3 \%)$, single $(59.8 \%)$, with a mean age between 18 and 40 years old (63.5\%), who declared themselves to be mixed race $(60.9 \%)$ and had 9 years of education or fewer (78.5\%). The prevalence of STI was $25.5 \%(90 / 353,95 \% \mathrm{CI}$ : 21.2\%-30.3\%), for HIV 4,0\% (14/353), syphilis 22,1\% (78/ $353)$ and hepatitis $\mathrm{B} 0,8 \%$ (3/353). It was verified that age (RP: 1.02, 95\% CI: 1.00-1.03), female sex (RP: 1.72, 95\% CI: 1.18-2.50), sex with members of the same sex (RP: 1.62, 95\% CI: 1.11-2.37) and sex with drug users (RP: 1.81, 95\% CI: 1.18-2.77) were statistically associated with STI positivity $(\mathrm{p} \leq 0.01)$.

Conclusion The results of this study show that life on the streets is a significant risk factor for exposure to STIs, and requires proactive health outreach. Street outreach offices are one effective public health strategy to access the homeless population and can act in the prevention and control of STIs.

\section{P3.119 THE EFFECT OF HSV-2 INFECTION ON SUBSEQUENT HIV ACQUISITION: AN UPDATED SYSTEMATIC REVIEW AND META-ANALYSIS}

${ }^{1}$ Katharine J Looker, ${ }^{2}$ Jocelyn AR Elmes, ${ }^{3}$ Sami L Gottlieb, ${ }^{4}$ Joshua T Schiffer, ${ }^{1}$ Peter Vickerman, ${ }^{1}$ Katherine ME Turner, ${ }^{2}$ Marie-Claude Boily. ${ }^{1}$ University of Bristol, Bristol, UK; ${ }^{2}$ Imperial College London, London, UK; ${ }^{3}$ World Health Organisation, Geneva, Switzerland; ${ }^{4}$ Fred Hutchinson Cancer Research Centre and University of Washington, Seattle, USA

\subsection{6/sextrans-2017-053264.354}

Introduction HIV and herpes simplex virus type 2 (HSV-2) infections cause a large burden of disease globally and are correlated epidemiologically through shared risk factors. There is also evidence for direct, biological interactions, with HSV-2 infection increasing HIV susceptibility (and vice versa). We update and expand on two previous systematic reviews of the association between HSV-2 infection and HIV acquisition published over a decade ago.

Methods We conducted a systematic review and random-effects meta-analysis of 56 longitudinal studies of the association between HSV-2 infection and HIV acquisition. We calculated pooled effect sizes using DerSimonian-Laird inverse-variance methods for the association between existing (prevalent) HSV2 infection and HIV seroconversion and the first-ever pooled effect sizes for new (incident) HSV-2 infection on HIV. We extended previous evaluations through detailed meta-regression and sub-group analyses.

Results The risk of acquiring HIV infection was approximately doubled in the presence of prevalent HSV-2 infection, and higher among general populations $(\mathrm{aRR}=2.7$; 95\% CI 2.2-3.4, $\mathrm{n}=24$ ) than higher-risk populations such as commercial sex workers and men who have sex with men $(\mathrm{aRR}=1.7$; 95\% CI 1.4-2.1, $\mathrm{n}=25$ ). Incident HSV-2 infection was associated with the highest risk of acquiring HIV: up to aRR=4.7 $(95 \% \mathrm{CI}$ $2.2-10.1, n=6)$. Adjustment for confounders was often incomplete, but this did not meaningfully influence results in subgroup analysis. Substantial heterogeneity across study estimates was explained by risk group, timing of HSV-2 infection, and world region.

Conclusion There is good evidence for a direct effect of HSV2 infection on HIV acquisition risk. This has important implications for managing individuals diagnosed with genital herpes, 
given the greater risk of acquiring HIV, especially for those newly infected. Interventions targeted against HSV-2, such as new HSV vaccines, have the potential for an additional benefit against HIV, which could be substantial particularly in regions where co-infections are abundant.

\section{P3.120 PREVALENCE OF UREAPLASMA UREALYTICUM IN URINE OF MEN ATTENDING A SEXUALLY TRANSMITTED DISEASE CLINIC}

Kenneth Fife, James A Williams, Sarah Fortney, Brahim Qadadri, Aaron Ermel. Indiana University School of Medicine, Indianapolis, USA

\subsection{6/sextrans-2017-053264.355}

Introduction Ureaplasma urealyticum (UU) is probably one of the causes of non-gonococcal, non-chlamydial urethritis in men and post-partum endometritis in women. The epidemiology of UU is currently unclear because culture isolation is difficult and molecular identification is limited to specialised laboratories. Testing for UU would be useful for surveillance, disease management, and epidemiology. This study assessed the prevalence of UU in men attending the local STD clinic by real-time PCR.

Methods A convenience sample of de-identified residual urine specimens from men attending an STD clinic was collected. Urine was placed into commercially available transport tubes and tested by PCR for Chlamydia trachomatis (CT), Neisseria gonorrhoeae (NG), and Trichomonas vaginalis (TV). The remaining residual processed specimen was tested for UU DNA using a previously published real-time PCR assay. Descriptive statistics were used to examine UU prevalence with age, and co-infection with CT, NG, and TV.

Results A total of 99 residual male urine specimens were available for testing. UU DNA was detected in 16/99 (16.2\%) of the specimens and was comparable to CT (14/97, 14.4\%), NG $(11 / 97,11.3 \%)$, and TV $(4 / 25,16.0 \%)$. Of the 16 UU positive specimens, co-infection with CT was observed in one (6.25\%), NG in one (6.25\%), and the remaining $14(87.5 \%)$ had no other infection identified. The mean age of those individuals positive for UU DNA was 32.4 (range 18-63) while the mean age of those infected with CT, NG, and TV was $29.8,28.3$, and 36.8 years old, respectively.

Conclusions The prevalence of UU in men attending an STD clinic is similar to that observed for CT, NG, and TV. This study was useful in order to gain a better understanding of $\mathrm{UU}$ in this population including the age of those positive for UU, co-infection status with other commonly identified pathogens, and as a means to confirm the feasibility of real-time PCR testing using residual processed specimens. More studies are needed to elucidate the significance of $\mathrm{UU}$ in this population.

\section{P3.121 VAGINAL DISCHARGE: EXPLORING DEMOGRAPHIC PROFILE, RISK FACTORS AND ALTERNATIVE METHOD OF RELIABLE DIAGNOSIS THROUGH SELF-COLLECTION OF VAGINAL SWABS}

Z Khan, A Bhargava, P Mittal, R Bharti, P Puri, N Khunger, M Bala. VMMC and Safdarjung Hospital, New Delhi, India

10.1136/sextrans-2017-053264.356
Introduction Vaginal discharge (VD), a common gynaecological complaint, is often associated with bacterial vaginosis (BV), vulvovaginal candidiasis (VVC) and Trichomonas vaginitis (TV). Prevention and control of these STIs/RTIs require understanding of demographic patterns and risk factors. Moreover, diagnosis in resource constrained settings can be facilitated using self-collected vaginal swabs, provided the reliability of the method is established. Aims were to establish the (1) association of sexual, demographic and hygienic practices for the above infections and (2) reliability of self-obtained over clinician-collected vaginal swabs for diagnosis.

Methods A total of 550 females aged 18-45 years (median: 32) attending two NACO designated STI/RTI clinics (Jan 2015-May 2016) with abnormal vaginal discharge were evaluated for relevant risk factors using a questionnaire. Swabs were self-collected by patients after instructions and subsequently by a physician under speculum examination. They were then examined by standard bedside tests, Gram staining, wet mount and culture.

Results BV, VVC and TV were observed in 79 (14.4\%), 144 $(26.2 \%)$ and $3(0.5 \%)$ patients respectively. VVC coexisted with BV in $58(10.5 \%)$ patients. C. albicans was isolated in $84(58.33 \%)$ VVC cases. Prevalence of VD was higher in rural $(\mathrm{p}>0.5)$ and illiterate $(\mathrm{p}>0.5)$ patients. BV was strongly associated with douching (odds ratio 8.26) and moderately with use of condoms (odds ratio 1.6), whereas VVC showed minimal association. Use of cloths instead of sanitary pads was also a risk factor for BV (odds ratio 1.3) and with a minimal impact on VVC. Highly concordant self vs physician-collected results established the reliability of self-collected samples with Cohen's Kappa values of 0.95 (BV), 0.99 (VVC) and 1.0 (TV).

Conclusion Avoiding douching, using condoms and sanitary pads help in reducing the risk of acquiring VD. Reliability of self-collected swabs for diagnosis shall go a long way in strengthening National STI/RTI Program, especially in resource constrained settings.

\section{P3.122 SIGNIFICANT DECREASE OF CD4+ T-CELLS BETWEEN RECENT AND LONG-TERM INFECTION IN HIV-1 SUBTYPES NON-B IN THE NORTHEAST BRAZIL}

${ }^{1}$ Kledoaldo Oliveira De Lima, 2 Élcio Leal, ${ }^{3}$ Ana Maria Salustiano Cavalcanti, ${ }^{3}$ Daniela Medeiros Salustiano, Heloísa Ramos Lacerda. ${ }^{1}$ Post-graduation in Tropical Medicine, Federal University of Pernambuco, Recife, PE, Brazil; ${ }^{2}$ Hospital das Clinicas, Federal University of Pernambuco, Recife, PE, Brazil; ${ }^{3}$ Institute of Biotechnology, Federal University of Pará, Belém, PA, Brazil; ${ }^{4}$ Sector of Virology, Central Laboratory of Public Health, Recife, PE, Brazil

\subsection{6/sextrans-2017-053264.357}

Introduction Immunoassays for detection of HIV-1 recent infection are important in guiding prevention policies in more affected groups and, especially, in the monitoring of antiretroviral resistance when performed in conjunction with genotyping assays. The objective of this study was to evaluate the frequency of individuals with HIV-1 recent infection and to relate infection status to viral load, CD4 $+\mathrm{T}$ cells count and viral subtype.

Methods One hundred and one samples from individuals diagnosed with HIV-1 were obtained from five Voluntary and Counselling Testing Centres (VCTs) in the state of Pernambuco (Northeast - Brazil), from 2007 to 2009, and tested by BEDCEIA Enzyme Immunoassay for determination of recent/longterm infection. Then, the HIV-1 pol region was sequenced 